\title{
Adult Ileo-Ileo-Caecal Intussusception: Case Report and Literature Review
}

\author{
Sanjeev Singhal, ${ }^{1}$ Anu Singhal, ${ }^{2}$ Pankaj K. Arora, ${ }^{1}$ \\ Rahul Tugnait, ${ }^{1}$ Bishwanath Tiwari, ${ }^{1}$ Pawan Malik,, ${ }^{1}$ Ankur Subhash Dhuria, ${ }^{1}$ \\ Vineet Varghese, ${ }^{1}$ Mriganka Deuri Bharali, ${ }^{1}$ Singh Chandrakant, ${ }^{1}$ Pushkar, ${ }^{1}$ \\ Vishnu Panwar, ${ }^{3}$ Amit Ballani, ${ }^{4}$ Neeti Gupta, ${ }^{5}$ and Vijay Kumar Ramteke ${ }^{6}$ \\ ${ }^{1}$ Department of General Surgery, Northern Railway Central Hospital, Basant Lane, New Delhi 110055, India \\ ${ }^{2}$ Department of Radiology, ESI Model Hospital and PGIMSR, Basaidarapur, Ring Road, New Delhi 110015, India \\ ${ }^{3}$ Department of Anaesthesiology, Northern Railway Central Hospital, Basant Lane, New Delhi 110055, India \\ ${ }^{4}$ Department of Radiology, Northern Railway Central Hospital, Basant Lane, New Delhi 110055, India \\ ${ }^{5}$ Department of Casualty and Obstetrics \& Gynaecology, Northern Railway Central Hospital, Basant Lane, New Delhi 110055, India \\ ${ }^{6}$ Medical Directorate, Indian Railways, Rail Bhawan, Rafi Marg, New Delhi 110001, India
}

Correspondence should be addressed to Anu Singhal,drsinghal_anu@yahoo.co.in

Received 8 October 2012; Accepted 18 November 2012

Academic Editors: K. Honma, K. W. Lobdell, J. M. Strzelczyk, and Y. Takami

Copyright (c) 2012 Sanjeev Singhal et al. This is an open access article distributed under the Creative Commons Attribution License, which permits unrestricted use, distribution, and reproduction in any medium, provided the original work is properly cited.

Adult intussusception is a rare entity accounting for only $5 \%$ of all intussusceptions and causes approximately $1 \%$ of all adult intestinal obstructions. Unlike paediatric intussusceptions which are usually idiopathic, there is usually a lead point pathology which might be malignant in up to $50 \%$ cases. We present an unusual case of adult intussusception which was not diagnosed on any investigation including computerized tomographic (CT) scan and magnetic resonance imaging (MRI). It was a case of ileoileo-cecal intussusception caused by a large lipoma $38 \mathrm{~mm} \times 43 \mathrm{~mm} \times 61 \mathrm{~mm}$. It was treated by emergency laparotomy for acute intestinal obstruction. A conservative resection with ileostomy was performed with good postoperative recovery.

\section{Introduction}

Intussusception means telescoping of a proximal segment of bowel (intussusceptum) into the lumen of the adjacent distal segment (intussuscipiens). Rarely, a distal segment of the bowel telescopes into the lumen of the adjacent proximal segment, which is known as retrograde intussusception [1]. Intussusception is a relatively common cause of intestinal obstruction in children but a rare and uncommon clinical entity in adults. Adult intussusception (AI) constitutes approximately $5 \%$ of all intussusceptions [2], and it accounts for $1-5 \%$ of all adult intestinal obstructions [2, 3]. Adult intussusception is usually caused by a tumor acting as the apex of the intussusception. In both small- and large-bowel intussusception, lipoma is the most common benign tumor [4].

\section{Case Report}

The patient was a 55-year-old male, employed as a manual laborer in Indian Railways. Patient presented in emergency with severe abdominal pain, multiple episodes of vomiting, abdominal distension, and obstipation. He had a history of recurrent attacks of colicky abdominal pain, nausea, and vomiting over the last 2 years. There was a history of hospitalization for occasional episodes of severe abdominal pain and distension and obstipation over this period. The frequency of these attacks had been progressively increasing over the last 2 years with the patient requiring weekly admission for the preceding two months before coming to our centre. There was history of loss of weight and appetite. There was no associated history of fever. There was no contributory past or family history. He was a chronic smoker of 80 packet 
years and a social alcoholic. He was investigated by CT scan of the abdomen and Thorax and MRI of the abdomen during his previous admissions and was diagnosed as a case of hiatus hernia with retroperitoneal fibrosis/diffuse lymphoproliferative disorder. CT chest showed nodularity indicative of infective pathology. However, these investigations were not available with the patient at the time of admission to our hospital. He was normotensive and afebrile but was having tachycardia and tachypnoea (pulse: 108/minute and respiratory rate: $22 /$ minute). His nutritional status appeared adequate. His general physical examination was essentially normal.

His systemic examination of chest, CVS, and CNS revealed no obvious abnormality. On abdominal examination he had a distended abdomen with stretched umbilicus. There were no visible scars. Abdomen was diffusely tender and guarded with no palpable organomegaly. Examination of external genitalia, hernial orifices and renal angles revealed no abnormality. On a digital rectal examination the rectum was empty and ballooned. A provisional diagnosis of acute intestinal obstruction with impending strangulation was made and confirmed on plain X-ray of the chest and abdomen erect and supine which showed grossly dilated small bowel loops and absence of any free intraperitoneal air. His haemogram, biochemical parameters, and urinanalysis done in emergency were within normal limits.

The patient was taken up for emergency laparotomy without any other investigations as clinically there was an impending strangulation. The abdomen was opened by a midline incision. There was a dilatation of small bowel till the terminal ileum where there was an ileo-ileo-caecal intussusception. The telescoping had started in the terminal ileum and gone through the ileo-caecal valve with the apex extending up to midascending colon (Figure 1). There was no other organomegaly lymphadenopathy or free fluid. The intussusception was reduced by gentle traction and retrograde pressure from the apex. There was a smooth nodule on the lateral wall of the terminal ileum at approximately $40 \mathrm{~cm}$ from IC junction (Figure 2). Good bowel viability was ensured. In view of emergency setting, unprepared bowel, lack of preoperative diagnosis, and absence of any lymphadenopathy/nodularity or free fluid it was decided to do a limited resection and bring out an ileostomy and mucous fistula. The ileo-caecal junction and valve were preserved. On resection and gross-examination there was a $38 \mathrm{~mm} \times$ $43 \mathrm{~mm} \times 61 \mathrm{~mm}$ nodule in the wall of ileum (Figure 3 ) which on histopathology was revealed to be a lipoma (Figure 4). The patient had an uneventful postoperative recovery.

\section{Discussion}

Since its first description in 1674 by Barbette [5] intussusception has been considered to be a disease of infancy and early childhood. Adult intussusception is distinct from pediatric intussusception. It is rare, the condition being found in less than 1 in 1300 abdominal operations and 1 in 100 patients operated for intestinal obstruction. The child to adult ratio is nearly $20: 1$ [6]. In contrast to intussusceptions in children

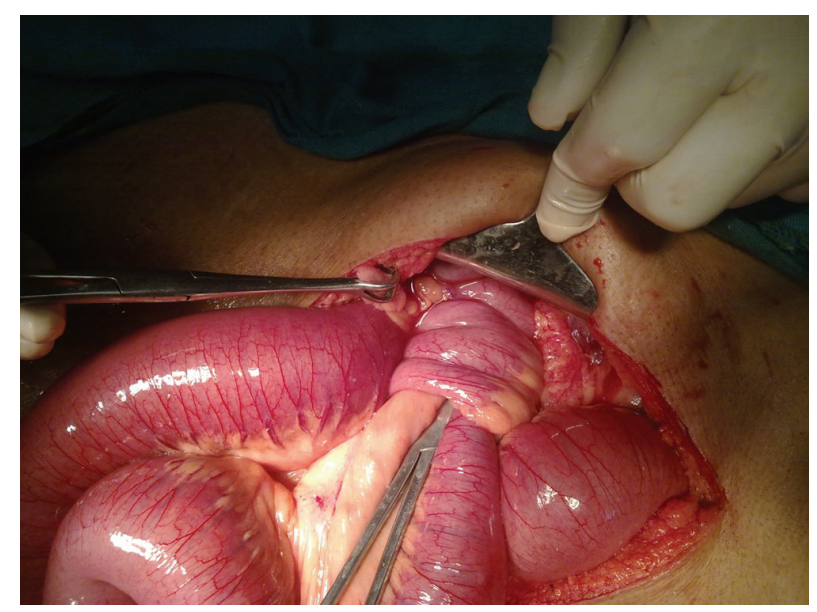

FIGURE 1: Terminal ileum telescoping into distal ileum and subsequently into ascending colon. Appendix is seen in normal position.

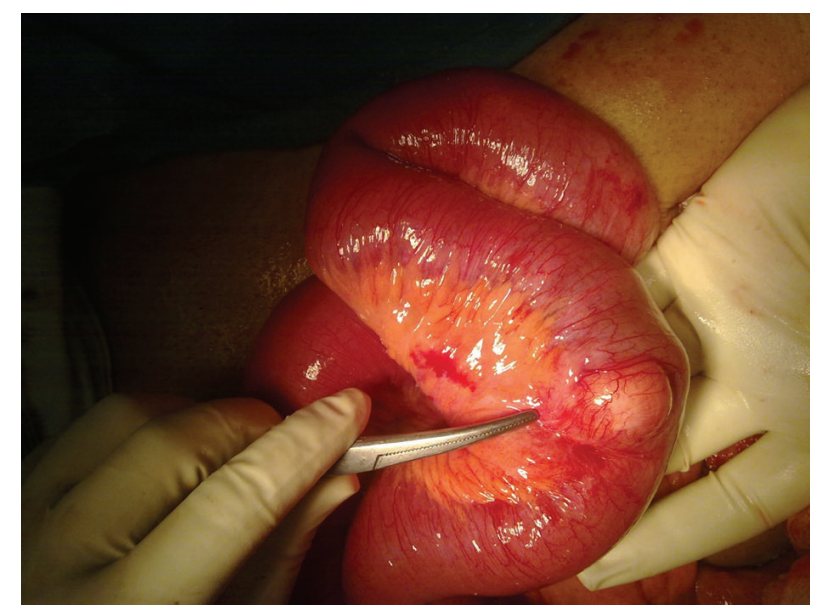

Figure 2: Externally visible tumor which served as a lead point. Note that the bowel is healthy, and mesenetry has no lymph nodes.

where nearly $80 \%$ are idiopathic, a demonstrable etiology is found in nearly $90 \%$ of cases in the adult population [7]. This necessitates resection in adults as against reduction in children.

Intussusceptions are classified according to location into: enteric, colonic, and ileocaecal or ileocolic [5]. Enteric and colonic intussusceptions are those that are confined to the small and large intestine, respectively. Ileocolic intussusceptions are defined as those in which ileum prolapses through the ileo-caecal valve into the colon, and these constitute $15 \%$ of all intussusceptions. The ileo-caecal valve and the appendix preserve their normal anatomical position, and the organic lesion is usually in the ileum [8]. Our case was one where a part of terminal ileum telescoped into the distal ileum and the whole intussusception then went through the ileo-caecal valve into the caecum and up to the ascending colon.

Small intestinal tumors are rare, accounting for 1-2\% of all gastrointestinal tract tumors [9]. Among these, benign 


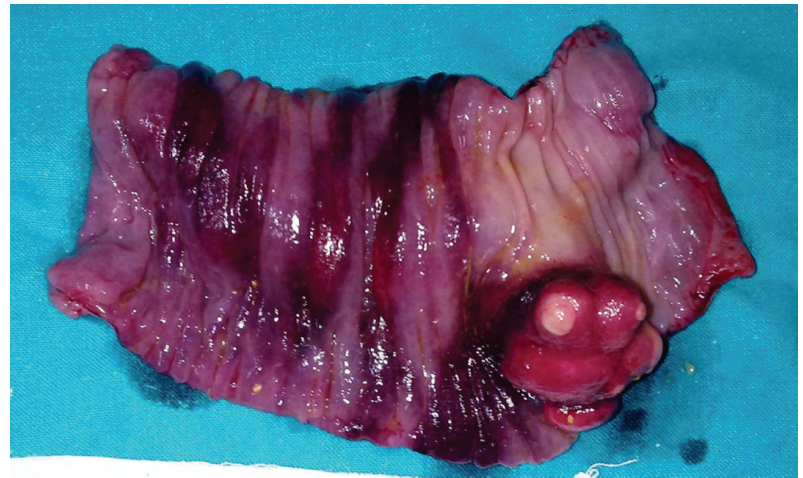

FIGURE 3: Specimen of resected terminal ileum cut section showing lobulated intraluminal growth.

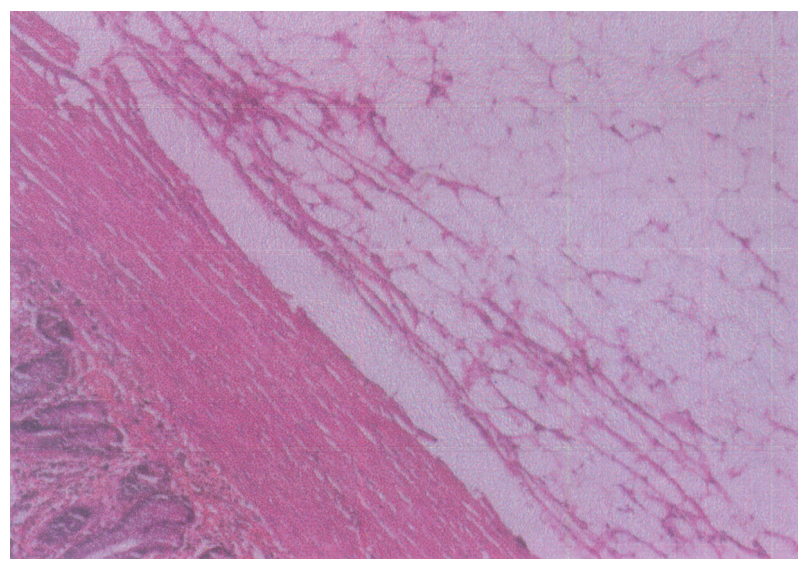

FIgURE 4: Histopathology showing lipoma.

tumors are still more rare and account for approximately $30 \%$ of all small bowel tumors [10]. The lipomas are rare benign tumors, representing $2.6 \%$ of nonmalignant tumors of the intestinal tract [11]. The incidence of intestinal lipomas has been reported between $0.15 \%$ and $4.4 \%$. Intestinal lipomas usually occur in older persons, with a slightly increased incidence in females [12, 13]. After gastrointestinal stromal tumors, lipomas constitute the second most common benign-tumor group [10]. Most occur in colon which constitutes from $65 \%$ to $75 \%$ of cases in comparison with small intestine which constitutes from $20 \%$ to $25 \%$ [14]. In the small bowel terminal ileum is the commonest site for lipomas [15].

Although they are usually asymptomatic, lipomas larger than $2 \mathrm{~cm}$ may cause bowel obstruction, intermittent nonspecific abdominal pain, diarrhea, or bleeding. Furthermore, some lipomas by forming a lead point may cause intussusception, as well $[10,16]$. Adult intussusceptions present with nonspecific obstructive symptoms like nausea, vomiting, and abdominal pain. Other symptoms may also be present such as melena, weight loss, fever, constipation, diarrhea, and abdominal mass [17]. In $20 \%$ to $50 \%$ of cases of adult intussusception, the etiologic agent is a malignancy [18].

Since the clinical picture is vague, varied, and nonspecific, preoperative diagnosis of adult intussusception is rare and essentially radiological. Plain skiagram of the abdomen may reveal features of acute intestinal obstruction [6]. On barium enema colonic lipomas appear as circular, ovoid, well demarcated, and smooth radiolucent masses (because of presence of fat). They show "squeeze sign" due to their fluctuation in size and shape [19].

Ultrasonography is often used to evaluate suspected intussusception as it is cheap, readily available, and noninvasive. The classic features include the "target and doughnut sign" on transverse view and the "pseudokidney sign" in longitudinal view. The major disadvantages are operator dependency and difficulty in image interpretation in presence of air, which is often present in cases of obstruction $[6,20]$. The preoperative diagnostic accuracy of ultrasonography is $78.5 \%$. In cases of palpable abdominal mass, the diagnostic accuracy of ultrasonography is even better $86.6 \%$ [6].

CT scan has been reported to be the most useful imaging technique, with a diagnostic accuracy of $58 \%-100 \%$ and a specificity of $57-71 \%[6,20]$. On CT, lipomas are seen as homogenous masses, well-circumscribed, ovoid, or round with sharp margins. In addition, they demonstrate characteristic attenuation values between -40 and $-120 \mathrm{HU}$ typical of the fatty composition [16]. The CT findings of intussusception are a mass-like lesion, including the inner intussusceptum, an eccentric fat density mass that represents the intussuscepted mesenteric fat, and the outer intussuscipiens, and this appears as a "target" or a "sausage" mass according to imaging plane [4]. CT is excellent in revealing the site, level, and cause of intestinal obstructions and in indicating possible bowel ischaemia. It can give additional information, such as metastasis or lymphadenopathy, which may indicate an underlying pathology [6]. Endoscopy can show a smooth yellow surface with a pedunculated or sessile base or either the "cushion sign" or "naked fat sign" [20].

In view of the uncertain aetiology and diagnosis and high incidence of malignancy (approaching 50\%), the treatment of intussusception in adults is invariably surgical resection. However, the extent of bowel resection and the manipulation of the intussuscepted bowel during reduction remain controversial $[7,8,15]$. In contrast to pediatric patients, where intussusception is primary and benign, preoperative reduction with barium or air is not suggested as a definite treatment for adults [7]. The theoretical risks of preliminary manipulation and reduction of an intussuscepted bowel include (1) intraluminal seeding and venous tumor dissemination, (2) perforation and seeding of microorganisms and tumor cells to the peritoneal cavity, and (3) increased risk of anastomotic complications of the manipulated friable and edematous bowel tissue [6-8]. Moreover, reduction should not be attempted if there are signs of inflammation or ischemia of the bowel wall and at age above 60 years [8]. However, several others believe that the risks are theoretical, and gentle reduction should be attempted in selected cases to avoid unnecessary resection of healthy bowel [15]. Endoscopic resection of colonic lipomatous polyps and laparoscopic resection of benign bowel tumors causing ileal and/or ileocolic intussusception has a role in very selected settings [6, $15]$. 


\section{Conclusion}

Adult intussusception is a rare entity which is distinct from paediatric cases in incidence, aetiology, and management. Ileo-colic intussusception is often caused by lead point pathology which can be a submucous lipoma but may be a malignant lesion thereby necessitating resection and histopathology. Our case had a long history, age of 55 years, and no bowel pathology on CT. Intraoperatively there was viable healthy bowel, and absence of any free fluid, lymph nodes, or nodules in liver. Therefore we attempted reduction by gentle traction and did a limited resection with ileostomy. In adult patients with long history and investigations and intraoperative findings favoring a benign pathology, it is possible to do avoid sacrificing unnecessary length of terminal ileum, more so where it is possible to save the ileo-caecal valve.

\section{Authors' Contribution}

All authors have contributed significantly to the paper in terms of clinical material, radiological diagnosis, surgical craft, and final preparation of the manuscript.

\section{Conflict of Interests}

There is no conflict of interests.

\section{Acknowledgment}

The study is cleared by the ethical committee of the hospital, and patients have given consent for the use of their clinical data for publication purpose.

\section{References}

[1] M. Chand, L. Bradford, and G. F. Nash, "Intussusception in colorectal cancer," Clinical Colorectal Cancer, vol. 7, no. 3, pp. 204-205, 2008.

[2] A. Zubaidi, F. Al-Saif, and R. Silverman, "Adult intussusception: a retrospective review," Diseases of the Colon and Rectum, vol. 49, no. 10, pp. 1546-1551, 2006.

[3] H. L. Laws and J. S. Aldrete, "Small bowel obstruction: a review of 465 cases," Southern Medical Journal, vol. 69, no. 6, pp. 733$734,1976$.

[4] B. Mehmet, T. Huseyin, C. A. Issam, Y. Erkan, and K. Ercan, "Ileocecal Intussusception due to a Lipoma in an Adult," Case Reports in Surgery, vol. 2012, Article ID 684298, 4 pages, 2012.

[5] K. K. Se, O. K. Jae, K. K. Oh et al., "A rare ileal intussusception caused by a Lipoma of the ileum," Journal of the Korean Surgical Society, vol. 77, pp. 59-63, 2009.

[6] K. G. Rakesh, S. A. Chandra, Y. Rohit, B. Amir, and L. S. Panna, "Intussusceptions in adults: a retrospective interventional series of cases," Health Renaissance, vol. 8, no. 3, pp. 158-165, 2010.

[7] A. Marinis, A. Yiallourou, L. Samanides et al., "Intussusception of the bowel in adults: a review," World Journal of Gastroenterology, vol. 15, no. 4, pp. 407-411, 2009.

[8] B. Hany and D. Samer, "Ileal lipoma-a rare cause of ileocolic intussusception in adults: case report and literature review,"
World Journal of Gastrointestinal Surgery, vol. 3, no. 1, pp. 13$15,2011$.

[9] C. A. Good, "Tumors of the small intestine," The American Journal of Roentgenology, Radium Therapy, and Nuclear Medicine, vol. 89, pp. 685-705, 1963.

[10] T. Yao, "Primary small intestinal tumors," Stomach and Intestine, vol. 36, no. 7, p. 881, 2001.

[11] C. W. Mayo, R. J. G. Pagtalunan, and D. J. Brown, "Lipoma of the alimentary tract," Surgery, vol. 53, no. 5, pp. 598-603, 1963.

[12] G. Ghidirim, I. Mishin, E. Gutsu, I. Gagauz, A. Danch, and S. Russu, "Giant submucosal lipoma of the cecum. Report of a case and review of literature," Romanian Journal of Gastroenterology, vol. 14, no. 4, pp. 393-396, 2005.

[13] S. Boyce and Y. P. Khor, "A colonic submucosal lipoma presenting with recurrent intestinal obstruction attacks," BMJ Case Report, June 2009.

[14] S. W. Ashley and S. A. Wells Jr., "Tumors of the small intestine," Seminars in Oncology, vol. 15, no. 2, pp. 116-128, 1988.

[15] I. Akagi, M. Miyashita, M. Hashimoto, H. Makino, T. Nomura, and T. Tajiri, "Adult intussusception caused by an intestinal lipoma: report of a case," Journal of Nippon Medical School, vol. 75, no. 3, pp. 166-170, 2008.

[16] H. Akyýldýz, I. Biri, A. Akcan, C. Küçük, and E. Sözüer, "Ileal lipoma: case report," Erciyes Medical Journal, vol. 33, no. 1, pp. 083-086, 2011.

[17] T. Azar and D. L. Berger, "Adult intussusception," Annals of Surgery, vol. 226, no. 2, pp. 134-138, 1997.

[18] D. F. Briggs, J. Carpathios, and R. W. Zollinger, "Intussusception in adults," The American Journal of Surgery, vol. 101, no. 1, pp. 109-113, 1961.

[19] M. Shramana, K. Vibha, K. D. Kajal, G. Parul, and K. Nita, "Lipomatous polyp presenting with intestinal intussusception in adults: report of four cases," Gastroenterology Research, vol. 3, no. 5, pp. 229-231, 2010.

[20] A. M. Minaya Bravo, C. Vera Mansilla, F. Noguerales Fraguas, and F. J. Granell Vicent, "Ileocolic intussusception due to giant ileal lipoma: review of literature and report of a case," International Journal of Surgery Case Reports, vol. 3, pp. 382-384, 2012. 


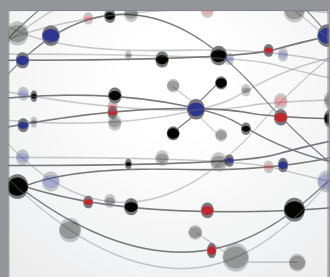

The Scientific World Journal
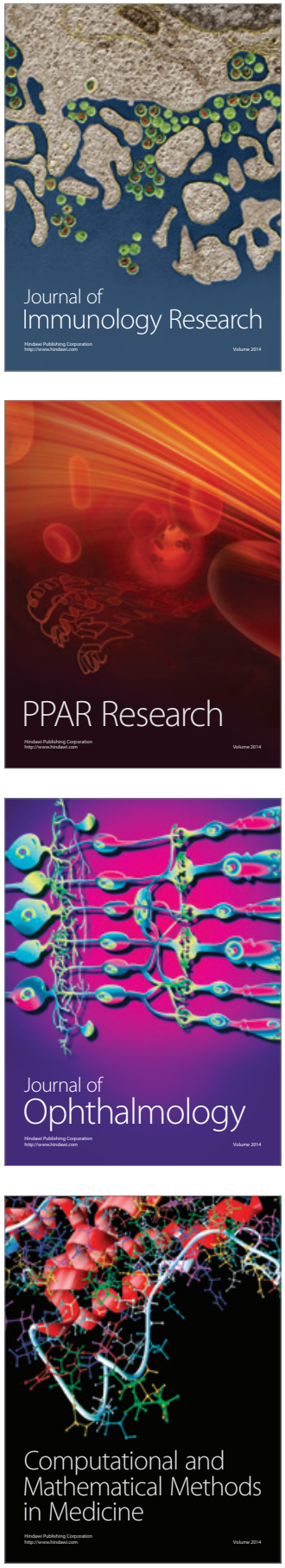

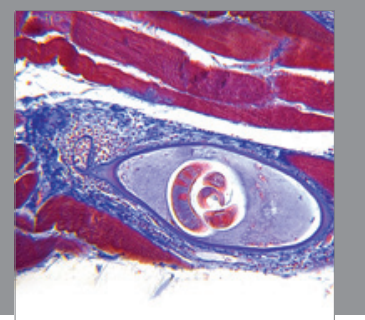

Gastroenterology

Research and Practice
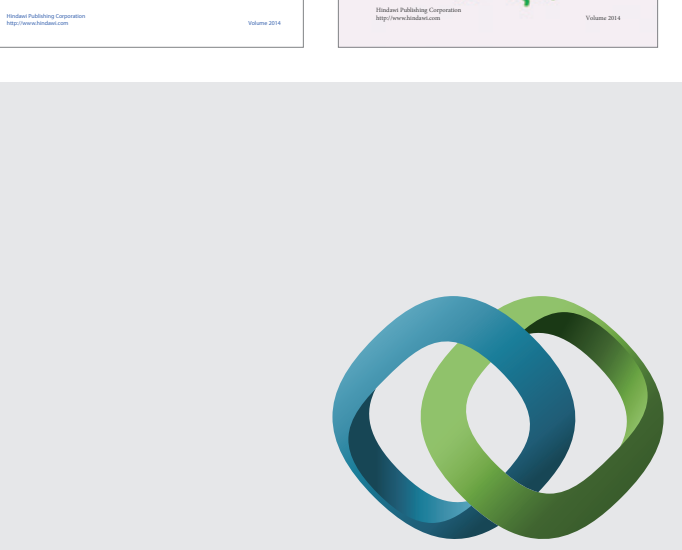

\section{Hindawi}

Submit your manuscripts at

http://www.hindawi.com
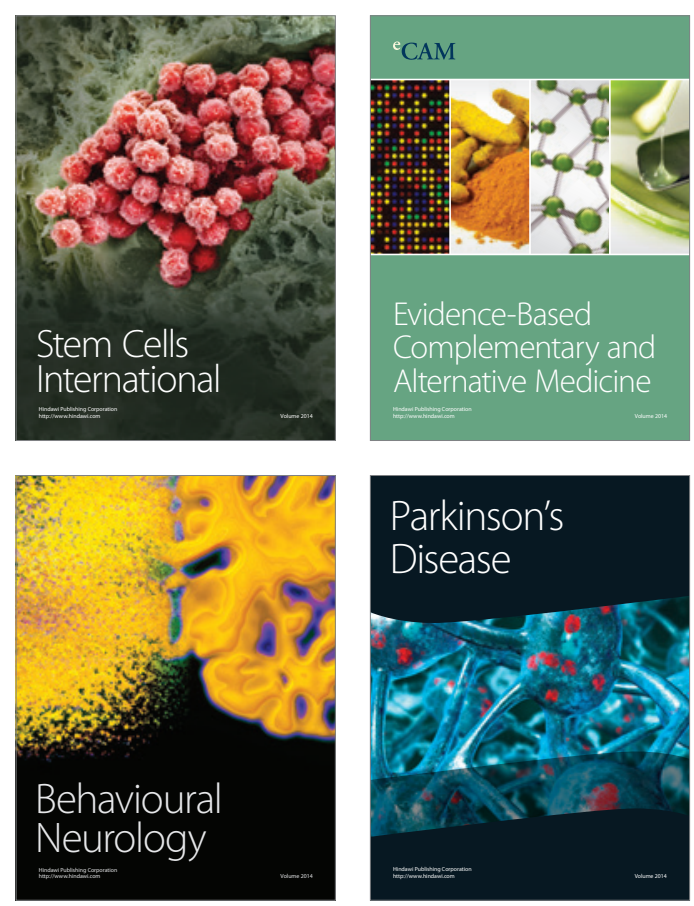

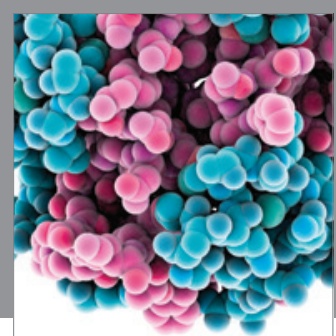

Journal of
Diabetes Research

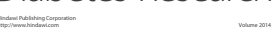

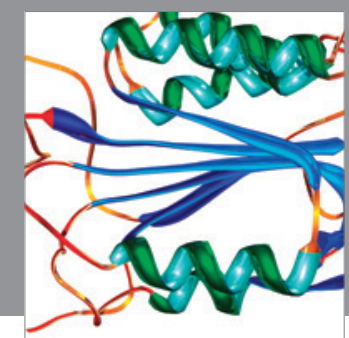

Disease Markers
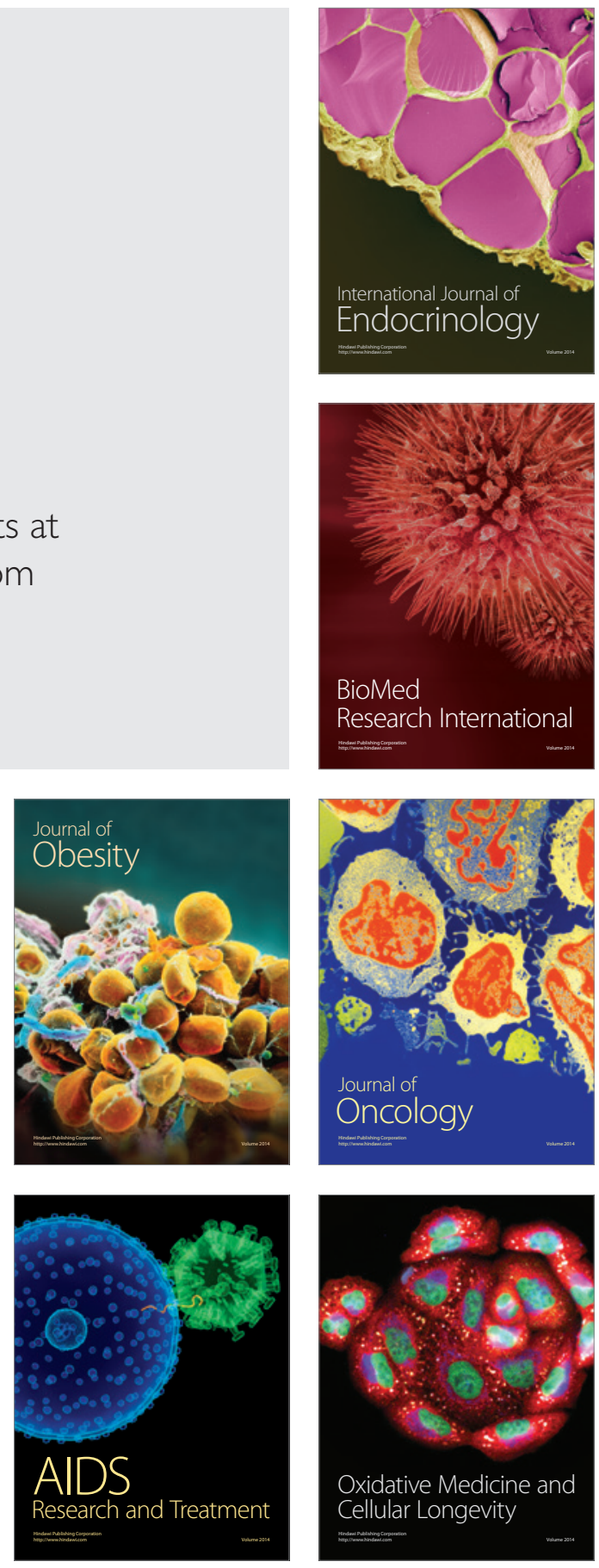